\title{
Multiplicity of positive solutions to second-order singular differential equations with a parameter
}

\author{
Shengjun $\mathrm{Li}^{1,2^{*}}$, Fang-fang $\mathrm{LiaO}^{3}$ and Hailong $\mathrm{Zhu}^{4}$
}

\section{"Correspondence:}

shjli626@126.com

${ }^{1}$ College of Science, Hohai

University, Nanjing, 210098, China

${ }^{2}$ Department of Mathematics,

Hainan University, Haikou, 570228,

China

Full list of author information is

available at the end of the article

\begin{abstract}
We study the existence and multiplicity of positive periodic solutions for second-order nonlinear damped differential equations by combing the analysis of positiveness of the Green function for a linear damped equation. Our nonlinearity may be singular in its dependent variable. The proof of the main result relies on the Guo-Krasnosel'skii fixed point theorem on compression and expansion of cones.
\end{abstract}

MSC: 34B16; 34C25

Keywords: positive solutions; singular; Guo-Krasnosel'skii fixed point theorem

\section{Introduction}

In this paper, we study the existence and multiplicity of positive $T$-periodic solutions for the following second-order singular differential equation:

$$
-x^{\prime \prime}+a(t) x^{\prime}+b(t) x=\lambda f\left(t, x, x^{\prime}\right), \quad 0 \leq t \leq T,
$$

where $\lambda>0$ is a positive parameter, $a, b \in \mathbb{C}(\mathbb{R} / T \mathbb{Z}, \mathbb{R})$ and the nonlinearity $f \in((\mathbb{R} / T \mathbb{Z}) \times$ $(0,+\infty) \times \mathbb{R}, \mathbb{R})$. In particular, the nonlinearity may change sign and have a repulsive singularity at $x=0$, which means that

$$
\lim _{x \rightarrow 0^{+}} f(t, x, y)=+\infty \quad \text { uniformly in }(t, y) \in \mathbb{R}^{2} .
$$

Equation (1.1) is a particular case of a more general class of Sturm equations of the type

$$
-\left[p(t) x^{\prime}\right]^{\prime}+q(t) x=f(t, x)
$$

where $p$ is a strictly positive absolutely continuous function. Such equations, even in the case $p \equiv 1$, where they are referred to as being of Schrödinger or Klein-Gordon type, appear in many scientific areas including quantum field theory, gas dynamics, fluid mechanics and chemistry.

Electrostatic or gravitational forces are the most important examples of singular interactions. During the last few decades, the study of the existence of positive solutions for singular differential equations has deserved the attention of many researchers [1-7]. Some

\section{望 Springer}

(02014 Li et al.; licensee Springer. This is an Open Access article distributed under the terms of the Creative Commons Attribution License (http://creativecommons.org/licenses/by/2.0), which permits unrestricted use, distribution, and reproduction in any medium, provided the original work is properly cited. 
strong force conditions introduced by Gordon [8] are standard in the related earlier works $[6,7,9]$. Compared with the case of strong singularity, the study of the existence of periodic solutions under the presence of a weak singularity is more recent, but has also attracted many researchers $[2,3,10,11]$. In particular, the degree theory [6, 7], the method of upper and lower solutions [11, 12], Schauder's fixed point theorem $[2,10]$, some fixed point theorems in cones for completely continuous operators [13-16] and a nonlinear LeraySchauder alternative principle [17-19] are the most relevant tools.

However, singular differential equation (1.1), in which the nonlinearity is dependent on the derivative and does not require $f$ to be nonnegative, has not attracted much attention in the literature. There are not so many existence results for (1.1) even when the nonlinearity is independent of the derivative. In this paper, we try to fill this gap and establish the existence of positive $T$-periodic solutions of (1.1) using the Guo-Krasnosel'skii fixed point theorem on compression and expansion of cones, which has been used to study positive solutions for systems of ordinary, functional differential equations [14-16]. We remark that it is sufficient to prove that $T: K \cap\left(\tilde{\Omega}_{2} \backslash \Omega_{1}\right) \rightarrow K$ is continuous and completely continuous in Lemma 3.2 (Section 3). This point is essential and advantageous.

As mentioned above, this paper is mainly motivated by the recent paper $[14,16]$. The aim of this paper is to study the multiplicity of positive solutions to (1.1). It is proved that such a problem has at least two positive solutions under reasonable conditions (see Theorem 3.5). And the remaining part of this paper is organized as follows. In Section 2, we find the Green function of the linear damped equation

$$
-x^{\prime \prime}+a(t) x^{\prime}+b(t) x=0, \quad 0 \leq t \leq T,
$$

subject to periodic boundary conditions

$$
x(0)=x(T), \quad x^{\prime}(0)=x^{\prime}(T),
$$

and prove its positiveness. The fact is very crucial to our arguments. Moreover, the onesigned property of the Green function implies that a maximum principle and an antimaximum principle hold for the corresponding linear differential equations subject to various boundary conditions, which is an important topic in differential equations (see [20, 21]). In Section 3, by employing the Guo-Krasnosel'skii fixed point theorem, we prove the existence of twin positive solutions for (1.1) under the positiveness of the Green function associated with (1.2)-(1.3). To illustrate the new results, some applications are also given.

\section{The Green function and its positiveness}

In this section, we consider the nonhomogeneous equation

$$
-x^{\prime \prime}+a(t) x^{\prime}+b(t) x=h(t), \quad 0 \leq t \leq T .
$$

We say that (1.2)-(1.3) is nonresonant if its unique $T$-periodic solution is the trivial one. When (1.2)-(1.3) is nonresonant, as a consequence of Fredholm's alternative, equation (2.1) admits a unique $T$-periodic solution which can be written as

$$
x(t)=\int_{0}^{T} G(t, s) h(s) d s,
$$


where $h \in \mathbb{C}(\mathbb{R} / T \mathbb{Z}), G(t, s)$ is the Green function of (2.1), associated with (1.3), and we will prove its positiveness. Throughout this paper, we assume that the following condition is satisfied:

(H) $a(t), b(t)$ are continuous functions and $b(t)>0$ for all $t \in[0, T]$.

Lemma 2.1 Let $K(s, \tau):[0, T] \times[0, T] \rightarrow[0,+\infty)$ be a continuous function. Then, for any nonnegative continuous function $\varphi(t)$ defined on $[0, T]$, the integral equation

$$
x(t)=\varphi(t)+\int_{0}^{t} \int_{0}^{s} K(s, \tau) x(\tau) d \tau d s, \quad t \in[0, T]
$$

has a unique solution $x(t)$, which is continuous on $[0, T]$ and satisfies the following inequality:

$$
x(t) \geq \varphi(t), \quad t \in[0, T] .
$$

Proof We solve equation (2.2) by the method of successive approximations. Let

$$
x_{0}(t)=\varphi(t), \quad x_{n}(t)=\int_{0}^{t} \int_{0}^{s} K(s, \tau) x_{n-1}(\tau) d \tau d s, \quad n=1,2, \ldots
$$

Take

$$
\alpha_{1}=\max _{0 \leq t \leq T} \varphi(t), \quad \beta_{1}=\max _{0 \leq s, \tau \leq T} K(s, \tau) .
$$

One can easily verify that

$$
0 \leq x_{n}(t) \leq \frac{\alpha_{1} \beta_{1}^{n}}{(2 n) !} t^{2 n}, \quad n=1,2, \ldots
$$

which implies that the series $\sum_{n=0}^{\infty} x_{n}(t)$ converges uniformly with respect to $t \in[0, T]$. Obviously, $x(t)=\sum_{n=0}^{\infty} x_{n}(t)$ is a continuous solution of (2.2). Moreover, inequality (2.3) holds because $\varphi(t)$ and $K(s, \tau)$ are nonnegative functions.

Next we prove the uniqueness. To do so, we first show that the solution of (2.2) is unique on $\left[t_{1}, t_{2}\right] \subset[0, T]$ with $t_{2}<\sqrt{2 / \beta_{1}}$. Then the uniqueness of the solution on $[0, T]$ is direct using the continuation property.

On the contrary, suppose that (2.2) has two solutions $x_{1}$ and $x_{2}$ on $\left[t_{1}, t_{2}\right]$. Then, for $t \in\left[t_{1}, t_{2}\right]$, we have

$$
\begin{aligned}
\left|x_{1}(t)-x_{2}(t)\right| & =\left|\int_{0}^{t} \int_{0}^{s} K(s, \tau)\left(x_{1}(\tau)-x_{2}(\tau)\right) d \tau d s\right| \\
& \leq \int_{0}^{t} \int_{0}^{s} K(s, \tau)\left|x_{1}(\tau)-x_{2}(\tau)\right| d \tau d s \\
& \leq \max _{t \in\left[t_{1}, t_{2}\right]}\left|x_{1}(t)-x_{2}(t)\right| \beta_{1} t^{2} / 2 \\
& <\max _{t \in\left[t_{1}, t_{2}\right]}\left|x_{1}(t)-x_{2}(t)\right| .
\end{aligned}
$$

Hence it follows that $x_{1}(t)=x_{2}(t)$ for all $t \in\left[t_{1}, t_{2}\right]$. 
Let us denote by $u(t)$ and $v(t)$ the solutions of (1.2) satisfying the initial conditions

$$
u(0)=1, \quad u^{\prime}(0)=0 ; \quad v(0)=0, \quad v^{\prime}(0)=1 .
$$

Lemma $2.2 u(t)$ and $v(t)$ satisfy the following integral equations:

$$
\begin{aligned}
& u(t)=1+\int_{0}^{t} \int_{0}^{s} b(\tau) \exp \left(\int_{\tau}^{s} a(r) d r\right) u(\tau) d \tau d s, \\
& v(t)=\int_{0}^{t} \exp \left(\int_{0}^{s} a(r) d r\right) d s+\int_{0}^{t} \int_{0}^{s} b(\tau) \exp \left(\int_{\tau}^{s} a(r) d r\right) v(\tau) d \tau d s .
\end{aligned}
$$

Proof Since $u(t)$ is a solution of (1.2), we have

$$
\left(u^{\prime}(t) \exp \left(-\int_{0}^{t} a(r) d r\right)\right)^{\prime}=b(t) \exp \left(-\int_{0}^{t} a(r) d r\right) u(t) .
$$

Integrating (2.6) from 0 to $t$ and noticing $u^{\prime}(0)=0$, we obtain

$$
u^{\prime}(t)=\int_{0}^{t} b(\tau) \exp \left(\int_{\tau}^{t} a(r) d r\right) u(\tau) d \tau
$$

To obtain (2.4), we only need to integrate the above equality from 0 to $t$ and notice $u(0)=1$. In a similar way, we can prove (2.5).

Lemma 2.3 For the solution $x(t)$ of boundary value problem (2.1)-(1.3), the formula

$$
x(t)=\int_{0}^{T} G(t, s) h(s) d s, \quad t \in[0, T]
$$

holds, where

$$
\begin{aligned}
G(t, s)= & \frac{v(T)}{D} u(t) u(s)-\frac{u^{\prime}(T)}{D} v(t) v(s) \\
& + \begin{cases}\frac{v^{\prime}(T)-1}{D} u(t) v(s)-\frac{u(T)-1}{D} u(s) v(t), & 0 \leq s \leq t \leq T, \\
\frac{v^{\prime}(T)-1}{D} u(s) v(t)-\frac{u(T)-1}{D} u(t) v(s), & 0 \leq t \leq s \leq T,\end{cases}
\end{aligned}
$$

is the Green function, the number $D$ is defined by $D=u(T)+v^{\prime}(T)-2$.

Proof It is easy to see that the general solution of equation (2.1) has the form

$$
x(t)=\alpha_{2} u(t)+\beta_{2} v(t)+\int_{0}^{t}(u(t) v(s)-u(s) v(t)) h(s) d s,
$$

where $\alpha_{2}$ and $\beta_{2}$ are arbitrary constants. Substituting this expression for $x(t)$ in boundary condition (1.3), we can obtain

$$
\begin{aligned}
& \alpha_{2}=\alpha_{2} u(T)+\beta_{2} v(T)+u(T) \int_{0}^{T} v(s) h(s) d s-v(T) \int_{0}^{T} u(s) h(s) d s, \\
& \beta_{2}=\alpha_{2} u^{\prime}(T)+\beta_{2} v^{\prime}(T)+u^{\prime}(T) \int_{0}^{T} v(s) h(s) d s-v^{\prime}(T) \int_{0}^{T} u(s) h(s) d s .
\end{aligned}
$$

After not very complicated calculations, we can get (2.7) and (2.8). 
Remark 2.4 As a direct application of Lemma 2.3, if $a(t)=0, b(t)=k^{2}>0$, then the Green function $G(t, s)$ of boundary value problem (2.1)-(1.3) has the form

$$
G(t, s)= \begin{cases}\frac{k^{k(t-s)}+e^{k(T-t+s)}}{2 k\left(e^{k T}-1\right)}, & 0 \leq s \leq t \leq T, \\ \frac{e^{k(s-t)}+e^{k(T+t-s)}}{2 k\left(e^{k T}-1\right)}, & 0 \leq t \leq s \leq T .\end{cases}
$$

Lemma 2.5 Assume that $(\mathrm{H})$ holds. Then the Green function $G(t, s)$ associated with (2.1)(1.3) is positive for all $s, t \in[0, T]$.

Proof Since $G(t, s)=G(s, t)$, it is enough to prove that $G(t, s)>0$ for $0 \leq s \leq t<T$. Recall that $u(t)$ and $v(t)$ satisfy integral equations (2.4) and (2.5). By condition (H) and Lemma 2.1, it follows that

$$
\begin{aligned}
& u(t) \geq 1, \quad v(t) \geq \int_{0}^{t} \exp \left(\int_{0}^{s} a(r) d r\right) d s>0, \quad t \in[0, T], \\
& u(T)>1, \quad v^{\prime}(T)>1 .
\end{aligned}
$$

Now from (2.9) we get $D=u(T)+v^{\prime}(T)-2>0$. Setting

$$
\begin{aligned}
& E_{1}(t, s)=u(s) v(t)-u(t) v(s), \\
& E_{2}(t, s)=(v(T) u(t)-u(T) v(t)) u(s)+\left(v^{\prime}(T) u(t)-u^{\prime}(T) v(t)\right) v(s),
\end{aligned}
$$

for $s \leq t$, we have

$$
G(t, s)=\frac{1}{D}\left(E_{1}(t, s)+E_{2}(t, s)\right) .
$$

Evidently, $E_{2}(T, 0)=0, E_{1}(s, s)=0$ for $s \in[0, T]$ holds. Let us now show that

$$
\begin{array}{ll}
E_{1}(t, s)>0 & \text { for } s \in[0, T) \text { and } t \in(s, T], \\
E_{2}(t, s)>0 & \text { for } s \in[0, T], t \in[s, T] \text { and }(t, s) \neq(T, 0) .
\end{array}
$$

To prove (2.10), we note that for fixed $s \in[0, T)$,

$$
\frac{\partial^{2} E_{1}(t, s)}{\partial t^{2}}=a(t) \frac{\partial E_{1}(t, s)}{\partial t}+b(t) E_{1}(t, s)
$$

and

$$
E_{1}(s, s)=0,\left.\quad \frac{\partial E_{1}(t, s)}{\partial t}\right|_{t=s}=1 .
$$

Hence it follows that for all $s \in(t, T]$, we have

$$
\begin{aligned}
E_{1}(t, s)= & \int_{0}^{t} \exp \left(\int_{0}^{s} a(r) d r\right) d s \\
& +\int_{0}^{t} \int_{0}^{\tau} b(\omega) \exp \left(\int_{\omega}^{\tau} a(r) d r\right) E_{1}(\omega, t) d \omega d \tau .
\end{aligned}
$$

Using Lemma 2.1, we get from (2.12) that $E_{1}(t, s)>0$ for all $t \in(s, T]$. 
Next, we prove (2.11), note that for fixed $s \in[0, T)$,

$$
\frac{\partial^{2} E_{2}(t, s)}{\partial t^{2}}=a(t) \frac{\partial E_{2}(t, s)}{\partial t}+b(t) E_{2}(t, s)
$$

and

$$
E_{2}(T, s)=v(s),\left.\quad \frac{\partial E_{2}(t, s)}{\partial t}\right|_{t=T}=-u(s) .
$$

Hence it follows that, for all $t \in[s, T]$, we have

$$
\begin{aligned}
E_{2}(t, s)= & v(s)+u(s) \int_{t}^{T} \exp \left(\int_{0}^{s} a(r) d r\right) d s \\
& +\int_{t}^{T} \int_{0}^{\tau} b(\omega) \exp \left(\int_{\omega}^{\tau} a(r) d r\right) E_{2}(\omega, t) d \omega d \tau .
\end{aligned}
$$

Again using Lemma 2.1, we get from (2.13) that $E_{2}(t, s)>0$ if $(t, s) \neq(T, 0)$, and the proof is completed.

Under hypothesis $(\mathrm{H})$, we always define

$$
A=\min _{0 \leq s, t \leq T} G(t, s), \quad B=\max _{0 \leq s, t \leq T} G(t, s), \quad \sigma=A / B .
$$

Thus $B>A>0$ and $0<\sigma<1$. When $a(t)=0, b(t)=k^{2}>0$, and a direct calculation shows that

$$
A=\frac{e^{k T / 2}}{k\left(e^{k T}-1\right)}, \quad B=\frac{1+e^{k T}}{2 k\left(e^{k T}-1\right)}, \quad \sigma=\frac{2 e^{k T / 2}}{1+e^{k T}}<1 .
$$

\section{Main results}

In this section, we state and prove the new existence results for (1.1). The proof is based on the following well-known fixed point theorem on compression and expansion of cones, which we state here for the convenience of the reader, after introducing the definition of a cone.

Definition 3.1 Let $X$ be a Banach space and let $K$ be a closed, nonempty subset of $X$. $K$ is a cone if

(i) $\alpha u+\beta v \in K$ for all $u, v \in K$ and all $\alpha, \beta>0$,

(ii) $u,-u \in K$ implies $u=0$.

We also recall that a compact operator means an operator which transforms every bounded set into a relatively compact set. Let us define the function $\omega(t)=\lambda \int_{0}^{T} G(t, s) d s$ and use $\|\cdot\|_{1}$ to denote the usual $L^{1}$-norm over $(0, T)$, by $\|\cdot\|$ we denote the supremum norm of $\mathbb{C}[0, T]$.

Lemma 3.2 [22] Let X be a Banach space and $K(\subset X)$ be a cone. Assume that $\Omega_{1}, \Omega_{2}$ are open subsets of $X$ with $0 \in \Omega_{1}, \bar{\Omega}_{1} \subset \Omega_{2}$, and let

$$
\mathcal{A}: K \cap\left(\bar{\Omega}_{2} \backslash \Omega_{1}\right) \rightarrow K
$$


be a completely continuous operator such that either

(i) $\|\mathcal{A} u\| \geq\|u\|, u \in K \cap \partial \Omega_{1}$ and $\|\mathcal{A} u\| \leq\|u\|, u \in K \cap \partial \Omega_{2}$; or

(ii) $\|\mathcal{A} u\| \leq\|u\|, u \in K \cap \partial \Omega_{1}$ and $\|\mathcal{A} u\| \geq\|u\|, u \in K \cap \partial \Omega_{2}$.

Then $\mathcal{A}$ has a fixed point in $K \cap\left(\bar{\Omega}_{2} \backslash \Omega_{1}\right)$.

Let $X=\mathbb{C}[0, T]$ and define

$$
K=\left\{x \in X: x(t) \geq 0 \text { and } \min _{0 \leq t \leq T} x(t) \geq \sigma\|x\|\right\}
$$

where $\sigma$ is as in (2.14).

One may readily verify that $K$ is a cone in $X$. Now, suppose that $F:[0, T] \times \mathbb{R} \times \mathbb{R} \rightarrow$ $[0, \infty)$ is a continuous function. Define an operator

$$
(\mathcal{A} x)(t)=\int_{0}^{T} G(t, s) F\left(s, x(s), x^{\prime}(s)\right) d s
$$

for $x \in X$ and $t \in[0, T]$.

Lemma 3.3 $\mathcal{A}: X \rightarrow K$ is well defined.

Proof Let $x \in X$, then we have

$$
\begin{aligned}
\min _{0 \leq t \leq T}(\mathcal{A} x)(t) & =\min _{0 \leq t \leq T} \int_{0}^{T} G(t, s) F\left(s, x(s), x^{\prime}(s)\right) d s \\
& \geq A \int_{0}^{T} F\left(s, x(s), x^{\prime}(s)\right) d s \\
& =\sigma B \int_{0}^{T} F\left(s, x(s), x^{\prime}(s)\right) d s \\
& \geq \sigma \max _{0 \leq t \leq T} \int_{0}^{T} G(t, s) F\left(s, x(s), x^{\prime}(s)\right) d s \\
& =\sigma\|\mathcal{A} x\| .
\end{aligned}
$$

This implies that $\mathcal{A}(X) \subset K$ and the proof is completed.

It is easy to prove.

Lemma 3.4 $\mathcal{A}$ is continuous and completely continuous.

Now we present our main result.

Theorem 3.5 Suppose that (1.1) satisfies (H). Furthermore, assume that

$\left(\mathrm{H}_{1}\right) f:[0, T] \times \mathbb{R}^{+} \times \mathbb{R} \rightarrow \mathbb{R}$ is continuous and there exists a constant $M>0$ such that

$$
F(t, x, y)=f(t, x, y)+M \geq 0 \quad \text { for all }(t, x, y) \in[0, T] \times \mathbb{R}^{+} \times \mathbb{R}
$$

$\left(\mathrm{H}_{2}\right) \lim _{x \rightarrow 0^{+}} f(t, x, y)=+\infty$ and $\lim _{x \rightarrow+\infty} f(t, x, y) / x=+\infty$ uniformly $(t, y) \in \mathbb{R}^{2}$.

Then (1.1) has at least two positive T-periodic solutions for sufficiently small $\lambda$. 
Proof To show that (1.1) has a positive solution, we should only show that

$$
-x^{\prime \prime}+a(t) x^{\prime}+b(t) x=\lambda F\left(t, x(t)-M \omega(t), x^{\prime}(t)-M \omega^{\prime}(t)\right)
$$

has a positive solution $x$ satisfying (1.3) and $x(t)>M \omega(t)$ for $t \in[0, T]$. If it is right, then $\phi(t)=x(t)-M \omega(t)$ is a solution of (1.1) since

$$
\begin{aligned}
-\phi^{\prime \prime} & (t)+a(t) \phi^{\prime}(t)+b(t) \phi(t) \\
& =-(x(t)-M \omega(t))^{\prime \prime}+a(t)(x(t)-M \omega(t))^{\prime}+b(t)(x(t)-M \omega(t)) \\
& =\lambda F\left(t, x(t)-M \omega(t), x^{\prime}(t)-M \omega^{\prime}(t)\right)-\lambda M \\
& =\lambda f\left(t, x(t)-M \omega(t), x^{\prime}(t)-M \omega^{\prime}(t)\right) \\
& =\lambda f\left(t, \phi(t), \phi^{\prime}(t)\right),
\end{aligned}
$$

where $-\omega^{\prime \prime}(t)+a(t) \omega^{\prime}(t)+b(t) \omega(t)=\lambda$ is used.

Problem (3.1)-(1.3) is equivalent to the following fixed point of the operator equation:

$$
x(t)=(\mathcal{A} x)(t),
$$

where $\mathcal{A}$ is a completely continuous operator defined by

$$
(\mathcal{A} x)(t)=\lambda \int_{0}^{T} G(t, s) F\left(s, x(s)-M \omega(s), x^{\prime}(s)-M \omega^{\prime}(s)\right) d s .
$$

Since $\lim _{x \rightarrow+\infty} \frac{f(t, x, y)}{x}=+\infty$, there exists $r_{1} \geq M T$ such that

$$
x \geq \sigma r_{1} \quad \Rightarrow \quad \frac{f(t, x, y)}{x} \geq \frac{1}{\sigma} .
$$

For $r>0$, let $\Omega_{r}=\{x \in K:\|x\|<r\}$ and note that $\partial \Omega_{r}=\{x \in K:\|x\|=r\}$.

First we show

$$
\|\mathcal{A} x\| \leq\|x\| \quad \text { for } x \in K \cap \partial \Omega_{r_{1}} .
$$

Let $h(t)=\max \left\{f\left(t, x, x^{\prime}\right): \frac{\sigma}{2} r_{1} \leq x \leq r_{1}\right\}$ and $\lambda^{*}=\min \left\{\sigma^{2} / 2 A, M T / B\|h\|_{1}\right\}$. For any $x \in$ $\partial \Omega r_{1}$ and $0<\lambda<\lambda^{*}$, we can verify that

$$
\begin{aligned}
x(t)-M \omega(t) & \geq \sigma\|x\|-M \omega(t)=\sigma r_{1}-M \omega(t) \\
& \geq \sigma r_{1}-\lambda M B T \geq \sigma r_{1}-\frac{\sigma r_{1}}{2}=\frac{\sigma r_{1}}{2} .
\end{aligned}
$$

Then we have

$$
\begin{aligned}
(\mathcal{A} x)(t) & =\lambda \int_{0}^{T} G(t, s) F\left(s, x(s)-M \omega(s), x^{\prime}(s)-M \omega^{\prime}(s)\right) d s \\
& \leq \lambda B \int_{0}^{T} F\left(s, x(s)-M \omega(s), x^{\prime}(s)-M \omega^{\prime}(s)\right) d s
\end{aligned}
$$




$$
\begin{aligned}
& \leq \lambda B\|h\|_{1} \\
& \leq r_{1}=\|x\| .
\end{aligned}
$$

This implies $\|\mathcal{A} x\| \leq\|x\|$.

In view of the assumption

$$
\lim _{x \rightarrow+\infty} \frac{f\left(t, x, x^{\prime}\right)}{x}=\lim _{x \rightarrow+\infty} \frac{F\left(t, x-M \omega, x^{\prime}-M \omega^{\prime}\right)}{x-M \omega^{\prime}}=+\infty,
$$

then there is $r_{2}>\sigma r_{2}>r_{1}$ such that

$$
\frac{F\left(t, x-M \omega, x^{\prime}-M \omega^{\prime}\right)}{x-M \omega} \geq \lambda^{-1} \sigma^{-1} A^{-1} T^{-1}, \quad x \geq \sigma r_{2} .
$$

Hence, we have

$$
F\left(t, x-M \omega, x^{\prime}-M \omega^{\prime}\right) \geq \lambda^{-1} A^{-1} T^{-1} r_{2}, \quad x \geq \sigma r_{2} .
$$

Next, we show that

$$
\|\mathcal{A} x\| \geq\|x\| \quad \text { for } x \in K \cap \partial \Omega_{r_{2}} .
$$

To see this, let $x \in K \cap \partial \Omega r_{2}$, then

$$
\begin{aligned}
\|\mathcal{A} x\| & =\max _{0 \leq t \leq T}\left|\lambda \int_{0}^{T} G(t, s) F\left(s, x(s)-M \omega(s), x^{\prime}(s)-M \omega^{\prime}(s)\right) d s\right| \\
& \geq \lambda A \int_{0}^{T} F\left(s, x(s)-M \omega(s), x^{\prime}(s)-M \omega^{\prime}(s)\right) d s \\
& \geq \lambda A \int_{0}^{T} \lambda^{-1} A^{-1} T^{-1} r_{2} d s \\
& \geq r_{2}=\|x\| .
\end{aligned}
$$

It follows from Lemma 3.2 that $\mathcal{A}$ has a fixed point $\tilde{x}_{1}(t)$ such that $\tilde{x}_{1}(t) \in \bar{\Omega}_{r_{2}} \backslash \Omega_{r_{1}}$, which is a positive periodic solution of (3.1) for $\lambda<\lambda^{*}$ satisfying

$$
r_{1}<\left\|\tilde{x}_{1}\right\|<r_{2}
$$

So, equation (1.1) has a positive solution $x_{1}(t)=\tilde{x}_{1}(t)-M \omega(t) \geq \sigma r_{1}-\frac{\sigma M T}{2} \geq \frac{\sigma M T}{2}$.

On the other hand, since

$$
\lim _{x \rightarrow 0^{+}} f(t, x, y)=+\infty
$$

hence, there exists a positive number $0<r_{3}<r_{1}$ such that

$$
f(t, x, y)>0, \quad x \in \mathbb{R}^{+} \text {with } 0<x \leq r_{3}<\frac{\sigma M T}{2},
$$


problem (1.1)-(1.3) is equivalent to the following fixed point of the operator equation:

$$
x(t)=\left(\mathcal{A}^{\prime} x\right)(t),
$$

where $\mathcal{A}^{\prime}$ is a continuous and completely continuous operator defined by

$$
\left(A^{\prime} x\right)(t)=\lambda \int_{0}^{T} G(t, s) f\left(s, x(s), x^{\prime}(s)\right) d s
$$

And for any $\rho>0$, define

$$
\Delta(\rho)=\max \left\{f(t, x, y): x \in \mathbb{R}^{+}, \sigma \rho \leq x \leq \rho,(t, y) \in[0, T] \times \mathbb{R}\right\} .
$$

Furthermore, for any $x \in K \cap \partial \Omega r_{3}$, we have

$$
\begin{aligned}
\left(A^{\prime} x\right)(t) & =\lambda \int_{0}^{T} G(t, s) f\left(s, x(s), x^{\prime}(s)\right) d s \\
& \left.\leq \lambda B \int_{0}^{T} f\left(s, x(s), x^{\prime}(s)\right)\right) d s \\
& \leq \lambda B \Delta\left(r_{3}\right) T .
\end{aligned}
$$

Thus, from the above inequality, there exists $\lambda^{* *}$ such that

$$
\left\|\mathcal{A}^{\prime} x\right\|<\|x\| \quad \text { for } x \in \partial \Omega_{r_{3}}, 0<\lambda<\lambda^{* *} .
$$

Since $\lim _{x \rightarrow 0^{+}} f\left(t, x, x^{\prime}\right)=+\infty$, then there is a positive number $0<r_{4}<\sigma r_{3}<r_{3}$ such that

$$
f\left(t, x, x^{\prime}\right)>\gamma x, \quad x \in \mathbb{R}^{+} \text {with } 0<x \leq r_{4},
$$

where $\gamma$ satisfies $\lambda \gamma \sigma A T>1$.

If $x \in K \cap \partial \Omega r_{4}$, then

$$
\begin{aligned}
\left\|\mathcal{A}^{\prime} x\right\| & =\max _{0 \leq t \leq T}\left|\lambda \int_{0}^{T} G(t, s) f\left(s, x(s), x^{\prime}(s)\right) d s\right| \\
& \geq \lambda A \int_{0}^{T} f\left(s, x(s), x^{\prime}(s)\right) d s \\
& \geq \lambda A \int_{0}^{T} \gamma x d s \\
& \geq \lambda A \int_{0}^{T} \gamma \sigma\|x\| d s \\
& \geq\|x\| .
\end{aligned}
$$

It follows from Lemma 3.2 that $\mathcal{A}^{\prime}$ has a fixed point $x_{2}(t)$ such that $x_{2}(t) \in \bar{\Omega}_{r_{3}} \backslash \Omega_{r_{4}}$, which is a positive periodic solution of (1.1) for $\lambda<\lambda^{* *}$ satisfying

$$
r_{4}<\left\|x_{2}\right\|<r_{3}
$$


Noting that

$$
r_{4}<\left\|x_{2}\right\|<r_{3}<\frac{\sigma r_{1}}{2}<\left\|x_{1}\right\|<r_{2}
$$

we can conclude that $x_{1}$ and $x_{2}$ are the desired distinct positive periodic solutions of (1.1) for $\lambda<\min \left\{\lambda^{*}, \lambda^{* *}\right\}$.

Example Let the nonlinearity in (1.1) be

$$
f(t, x, y)=\left(1+|y|^{\gamma}\right)\left(c(t) x^{-\alpha}+d(t) x^{\beta}+e(t)\right), \quad 0 \leq t \leq T
$$

where $\alpha>0, \beta>1, \gamma \geq 0, c(t), d(t), e(x) \in \mathbb{C}[0, T]$. It is clear that $f(t, x, y)$ satisfies conditions $\left(\mathrm{H}_{1}\right),\left(\mathrm{H}_{2}\right)$. Then (1.1) has at least two positive $T$-periodic solutions for sufficiently small $\lambda$.

\section{Competing interests}

The authors declare that they have no competing interests.

\section{Authors' contributions}

All authors read and approved the final manuscript.

\section{Author details}

${ }^{1}$ College of Science, Hohai University, Nanjing, 210098, China. ${ }^{2}$ Department of Mathematics, Hainan University, Haikou, 570228, China. ${ }^{3}$ Nanjing College of Information Technology, Nanjing, 210046, China. ${ }^{4}$ School of Statistics and Applied Mathematics, Anhui University of Finance and Economics, Bengbu, 233030, China.

\section{Acknowledgements}

The authors express their thanks to the referees for their valuable comments and suggestions. This work is supported by the National Natural Science Foundation of China (Grant No. 11161017, No. 11301001, No. 11301139), Hainan Natural Science Foundation (Grant No.113001), Excellent Youth Scholars Foundation and the Natural Science Foundation of Anhui Province of PR China (No. 2013SQRL030ZD).

Received: 15 January 2014 Accepted: 28 April 2014 Published: 14 May 2014

\section{References}

1. Bravo, JL, Torres, PJ: Periodic solutions of a singular equation with indefinite weight. Adv. Nonlinear Stud. 10, 927-938 (2010)

2. Chu, J, Torres, PJ: Applications of Schauder's fixed point theorem to singular differential equations. Bull. Lond. Math. Soc. 39, 653-660 (2007)

3. Chu, J, Lin, X, Jiang, D, O'Regan, D, Agarwal, RP: Multiplicity of positive solutions to second order differential equations. Bull. Aust. Math. Soc. 73, 175-182 (2006)

4. Lazer, AC, Solimini, S: On periodic solutions of nonlinear differential equations with singularities. Proc. Am. Math. Soc. 99, 109-114 (1987)

5. Wang, F, Zhang, F, Ya, Y: Existence of positive solutions of Neumann boundary value problem via a convex functional compression-expansion fixed point theorem. Fixed Point Theory 11, 395-400 (2010)

6. Yan, P, Zhang, M: Higher order nonresonance for differential equations with singularities. Math. Methods Appl. Sci. 26, 1067-1074 (2003)

7. Zhang, M: Periodic solutions of equations of Ermakov-Pinney type. Adv. Nonlinear Stud. 6, 57-67 (2006)

8. Gordon, WB: Conservative dynamical systems involving strong forces. Trans. Am. Math. Soc. 204, 113-135 (1975)

9. del Pino, MA, Manásevich, RF: Infinitely many T-periodic solutions for a problem arising in nonlinear elasticity. J. Differ. Equ. 103, 260-277 (1993)

10. Franco, D, Torres, PJ: Periodic solutions of singular systems without the strong force condition. Proc. Am. Math. Soc. $136,1229-1236(2008)$

11. Rachunková, I, Tvrdý, M, Vrkoč, I: Existence of nonnegative and nonpositive solutions for second order periodic boundary value problems. J. Differ. Equ. 176, 445-469 (2001)

12. Bonheure, D, De Coster, C: Forced singular oscillators and the method of lower and upper solutions. Topol. Methods Nonlinear Anal. 22, 297-317 (2003)

13. Cabada, A, Cid, JA, Infante, G: New criteria for the existence of non-trivial fixed points in cones. Fixed Point Theory Appl. 2013, 125 (2013)

14. Wang, H: Positive periodic solutions of singular systems with a parameter. J. Differ. Equ. 249, 2986-3002 (2010)

15. Wang, H: On the number of positive solutions of nonlinear systems. J. Math. Anal. Appl. 281, 287-306 (2003) 
16. Wang, F, An, Y: Multiple positive doubly periodic solutions for a singular semipositone telegraph equation with a parameter. Bound. Value Probl. 2013, 7 (2013)

17. Jiang, D, Chu, J, Zhang, M: Multiplicity of positive periodic solutions to superlinear repulsive singular equations. J. Differ. Equ. 211, 282-302 (2005)

18. Li, S, Liang, L, Xiu, Z: Positive solutions for nonlinear differential equations with periodic boundary condition. J. Appl. Math. (2012). doi:10.1155/2012/528719

19. Zhu, H, Li, S: Existence and multiplicity results for nonlinear differential equations depending on a parameter in semipositone case. Abstr. Appl. Anal. (2012). doi:10.1155/2012/215617

20. Cabada, A, Cid, JA, Tvrdy, M: A generalized anti-maximum principle for the periodic one-dimensional $p$-Laplacian with sign-changing potential. Nonlinear Anal. 72, 3436-3446 (2010)

21. Campos, J, Mawhin, J, Ortega, R: Maximum principles around an eigenvalue with constant eigenfunctions. Commun. Contemp. Math. 10, 1243-1259 (2008)

22. Guo, D, Lakshmikanantham, V: Nonlinear Problems in Abstract Cones. Academic Press, New York (1988)

10.1186/1687-2770-2014-115

Cite this article as: Li et al:: Multiplicity of positive solutions to second-order singular differential equations with a

parameter. Boundary Value Problems 2014, 2014:115

\section{Submit your manuscript to a SpringerOpen ${ }^{\ominus}$ journal and benefit from:}

$\rightarrow$ Convenient online submission

Rigorous peer review

- Immediate publication on acceptance

- Open access: articles freely available online

- High visibility within the field

- Retaining the copyright to your article 